\title{
ARTICLE OPEN \\ E. coli Nissle 1917 is a safe mucosal delivery vector for a birch-grass pollen chimera to prevent allergic poly-sensitization
}

\author{
P. J. Sarate ${ }^{1}$, S. Heinl ${ }^{2}$, S. Poiret ${ }^{3}$, M. Drinić ${ }^{1}$, C. Zwicker ${ }^{1}$, I. Schabussova ${ }^{1}$, C. Daniel ${ }^{3}$ and U. Wiedermann ${ }^{1}$
}

\begin{abstract}
Allergic poly-sensitization affects a large number of allergic patients and poses a great challenge for their treatment. In this study we evaluated the effects of the probiotic Escherichia coli Nissle 1917 (EcN) expressing a birch and grass pollen allergen chimera 'Bet v 1, Phl p 1 and Phl p 5' (EcN-Chim) on allergy prevention after oral or intranasal application in poly-sensitized mice. In contrast to oral application, intranasal pretreatment with EcN-Chim prior to poly-sensitization led to a significant reduction of lung inflammation (eosinophils, IL-5, and IL-13 in bronchoalveolar lavage) along with suppressed levels of allergen-specific serum lgE. The suppression was associated with increased levels of allergen-specific IgA in lungs and serum IgG2a along with increased Foxp3, TGF- $\beta$, and IL-10 mRNA in bronchial lymph nodes. In vitro ECN induced high levels of IL-10 and IL-6 in both lung and intestinal epithelial cells. Importantly, using in vivo imaging techniques we demonstrated that intranasally applied EcN do not permanently colonize nose, lung, and gut and this strain might therefore be a safe delivery vector against allergy in humans. In conclusion, our data show that intranasal application of recombinant EcN expressing a multiallergen chimera presents a novel and promising treatment strategy for prevention of allergic poly-sensitization.
\end{abstract}

Mucosal Immunology (2019) 12:132-144; https://doi.org/10.1038/s41385-018-0084-6

\section{INTRODUCTION}

The burden of allergic diseases has increased significantly in Western countries over the last decades. Several factors such as changes in lifestyle, diet, increased hygiene and microbial dysbiosis have been suggested to be responsible for increased allergies. $^{1-3}$ Around $50-80 \%$ of mono-sensitized individuals exhibit poly-sensitization to different allergens with increasing age which significantly affects their quality of life. ${ }^{4}$ A common poly-sensitization profile in Europe is against birch and grass pollen allergens. ${ }^{5}$ The only curative treatment to reduce allergic symptoms in such poly-sensitized patients is subcutaneous or sublingual specific immunotherapy (SIT or SLIT, respectively). However, use of crude extracts of allergens in SIT/SLIT might induce side effects or even mount a certain risk of neosensitization to components in the extract. ${ }^{4,6}$ Furthermore, it has been shown that poly-sensitized patients are more difficult to successfully treat/tolerize than mono-sensitized individuals by conventional immunotherapy. ${ }^{5,6}$ Therefore, our allergy research has concentrated on the improvement of treatment strategies in poly-sensitized patients. $^{7-9}$

We previously have shown that poly-sensitization cannot be prevented by a mixture of allergens but that multiallergen constructs or polypeptides are needed for successful mucosal pretreatment. ${ }^{7,8}$ We first constructed polypeptides of birch and grass pollen allergens (Bet v 1, Phl p 1, Phl p 5) showing that airway inflammation was prevented by mucosal administration of these polypeptides. ${ }^{7,9}$ These polypeptides may however bear limitations for human use due to certain HLA restrictions. In this respect, allergen chimers including whole allergens as scaffold for other allergen peptides were constructed in our lab, the first one consisting of the major birch pollen allergen Bet v1 and the major grass pollen allergens $\mathrm{Phl} p 1$ and $\mathrm{Phl} \mathrm{p} 5$ and the second consisting of Bet $v 1$ and its homolog food allergens Api $g 1$ and Dau c.,10 However, as the in vivo efficacy of such constructs depends on the tolerogenic properties of all containing allergens/allergen peptides, addition of certain immunomodulating adjuvants/mucosal delivery systems might improve their function. ${ }^{11}$

Recently, probiotic bacteria have been shown to play an important role in reducing allergic sensitizations by restoring intestinal dysbiosis and modulating the immune system towards tolerance induction. ${ }^{12-14}$ In this regard, we have shown that intranasal application of Lactobacillus paracasei and Bifidobacterium longum during sensitization and challenge suppressed allergen-specific immune responses and airway inflammation in poly-sensitized mice. ${ }^{15}$ In another study we have shown that counter-regulatory Th1 responses can be induced more effectively by combined mucosal application of lactic acid bacteria with specific allergen in a murine model of birch pollen allergy. ${ }^{16}$ Probiotic bacteria such as Escherichia coli Nissle 1917 or lactic acid bacteria have been shown as effective antigen delivery systems for the prevention or treatment of various immune derived diseases in several mouse models. ${ }^{17}$ In humans, a recent phase 2

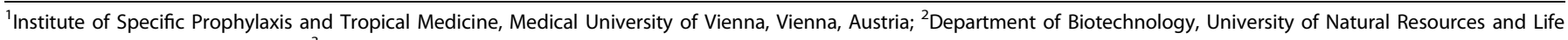

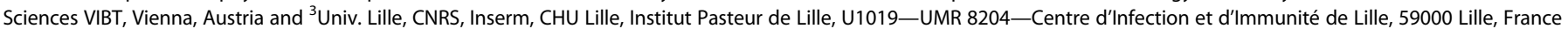
Correspondence: C. Daniel (catherine.daniel@ibl.cnrs.fr) or U. Wiedermann (ursula.wiedermann@meduniwien.ac.at)

These authors contributed equally: C. Daniel, U. Wiedermann.

Received: 2 March 2018 Revised: 27 July 2018 Accepted: 25 August 2018

Published online: 21 September 2018 
trial in ulcerative colitis patients has been successfully performed using recombinant EcN expressing feedback-resistant $N$-acetyl glutamate synthetase against hyperammonia. ${ }^{18}$ Similarly, also recombinant Lactococcus lactis have been tested against Crohn's disease (phase 1a trial) and oral mucositis in head and neck cancer patients (phase $1 \mathrm{~b}$ trial). ${ }^{19,20}$

Along these lines, we have previously shown that the major birch pollen allergen Bet $v 1$ can be expressed by lactic acid bacteria and mucosal application of these recombinant probiotic strains prevented allergic sensitization to birch pollen in adult conventional mice as well as in germ-free offspring derived from treated mothers. ${ }^{21,22}$ Thus, in the present study, we aimed to investigate if mucosal application of recombinant probiotic bacteria expressing a multiallergen chimera, i.e., birch-grass pollen chimera, can protect mice from the development of allergic poly-sensitization. For this purpose, the Bet $v$ 1, Phl p 1, and Phl p 5 chimera was cloned into the probiotic bacteria E. coli Nissle 1917 and this recombinant strain was applied by the intranasal or oral route to adult mice prior to allergic poly-sensitization and challenge. Our data provide novel insights into the effects and mechanisms of tolerance induction with the recombinant poly-allergenproducing probiotic bacteria as well as the migration pattern and persistence of probiotics applied via different mucosal routes.

\section{RESULTS}

E. coli Nissle 1917 induces high levels of IL-10 in naïve splenocytes and bone marrow-derived dendritic cells and activates both TLR2 and TLR4 signaling pathways

Probiotic bacterial strains L. plantarum NCIM8826, L. lactis MG1363 and E. coli Nissle 1917 (EcN) were compared for their intrinsic immunomodulatory properties. Naïve spleen cells and bone marrow-derived dendritic cells (BMDCs) were stimulated with $10^{7}$ colony-forming units/ml (CFU/ml) of $L$. plantarum, L. lactis, and EcN for $48 \mathrm{~h}$ and analyzed for cytokine response (Fig. 1). All three strains exhibited different immunomodulatory properties. In contrast to $L$. plantarum and L. lactis, EcN induced high levels of IL-10 and low levels of IL-12p70 in spleen cells (Fig. 1a). In BMDCs, a

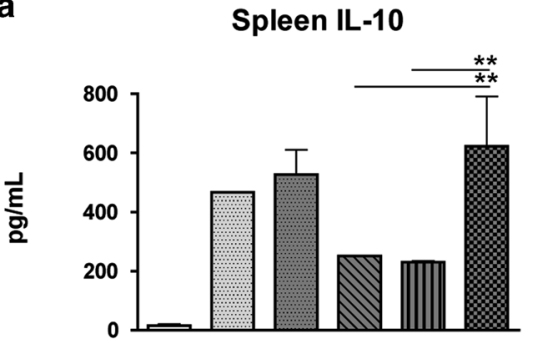

b

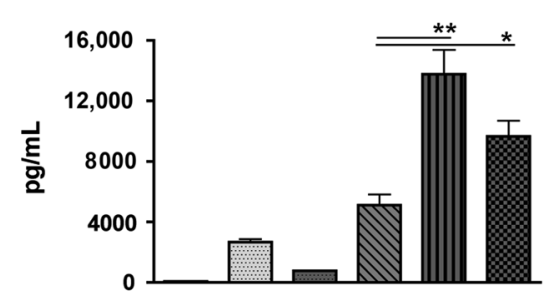

C

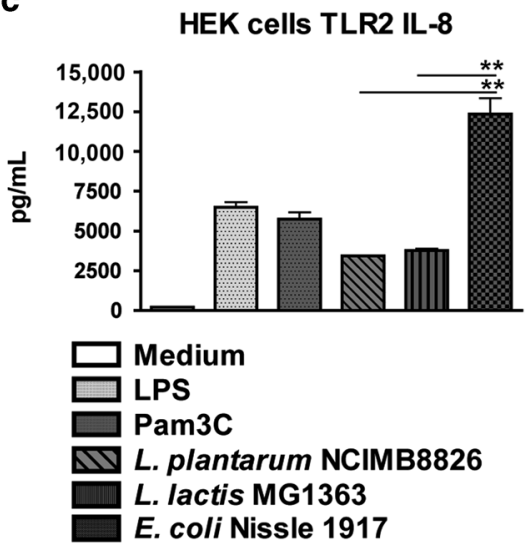

Spleen IL-12p70

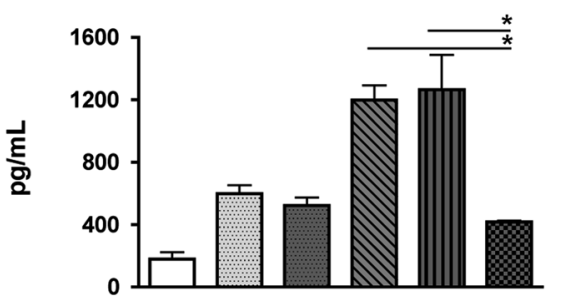

BMDC IL-12p70

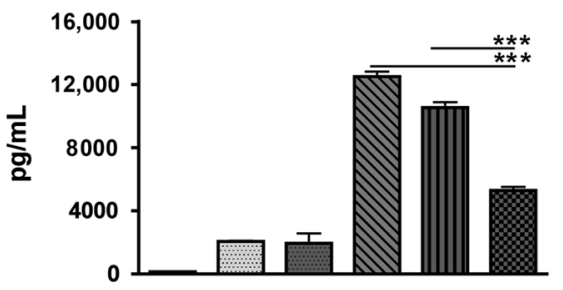

HEK cells TLR4 IL-8

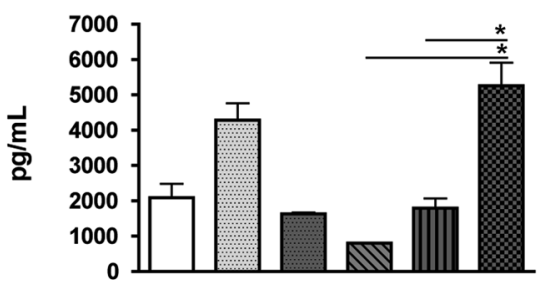

Spleen IL-5

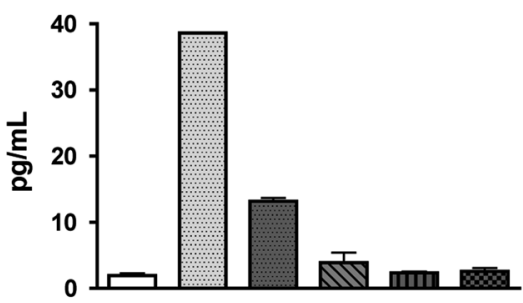

BMDC IL-5

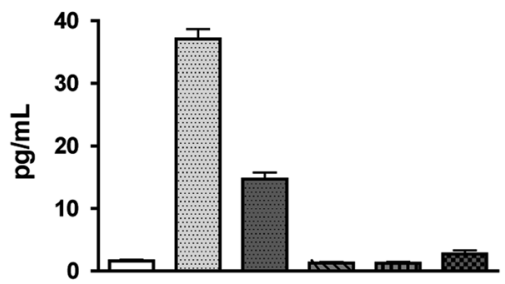

HEK cells IL-8

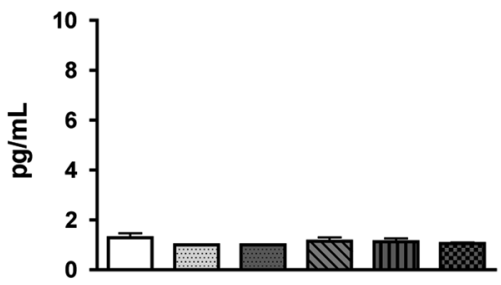

Fig. 1 E. coli Nissle 1917 induces high levels of IL-10 production in naïve splenocytes and bone marrow-derived dendritic cells (BMDCs) and activates both TLR2 and TLR4 signaling pathways. Spleen cells and BMDCs from wild-type mice as well as HEK293 cells expressing TLR2 or TLR4 were cultured with medium, LPS $(1 \mu \mathrm{g} / \mathrm{ml})$, Pam3CSK4 $(1 \mu \mathrm{g} / \mathrm{ml})$ as controls and $10^{7} \mathrm{CFU} / \mathrm{ml}$ of $L$. plantarum NCIM8826, L. lactis MG1363, and E. coli Nissle 1917. All cultures were incubated at $37^{\circ} \mathrm{C} 5 \% \mathrm{CO}_{2}$ for $48 \mathrm{~h}$. Supernatants from stimulated spleen cells and BMDCs were analyzed for cytokine production by ELISA. IL-5, IL-10, and IL-12p70 production in a spleen cells and b BMDCs. c Supernatants from bacteria stimulated HEK293 cells with or without TLR2 or TLR4 transfection were analyzed for IL-8 production. Three replicate cultures with cells from individual samples were measured. Data are representative of two independent experiments performed by using different batches of cell lines and bacterial culture. Error bars show mean \pm SEM. ${ }^{*} P<0.05,{ }^{*} P<0.01,{ }^{* * *} P<0.001$ by one-way ANOVA with Tukey's Multiple Comparison Test 
L. lactis and ECN stimulated higher levels of IL-10 production as compared to L. plantarum (Fig. 1b). Compared to L. plantarum and L. lactis, EcN induced significantly lower levels of IL-12p70. (Fig. 1b). None of the strains induced IL-5 production in spleen cells and BMDCs (Fig. 1a, b).

To assess which TLR signaling pathways were activated by the bacteria, HEK293 cells, with and without stable transfection with TLR2/CD14 or TLR4/MD-2/CD14 were incubated with the three strains. $L$. plantarum and $L$. lactis activated cells only via TLR2 signaling (measured by IL-8 production) whereas EcN showed activation of both TLR2 and TLR4-mediated signaling pathways (Fig. 1c). Stimulation of HEK293 cells without transfection with TLR2/4 with different bacteria did not lead to any IL-8 production, indicating that all three bacteria do not signal through other receptors than TLR2/4 (Fig. 1c).

Constitutive production of birch-grass pollen chimera by recombinant E. coli Nissle 1917

The recombinant birch-grass pollen chimera Phl p 5 - Bet v 1- Phl p 1, which was designed previously in our $\operatorname{lab}^{8}$, was successfully cloned in the lactococcal pNZ8148 vector and expressed in EcN under the constitutive lactate dehydrogenase promoter $\left(P_{\text {IdhL }}\right)$ with mCherry as a reporter gene (EcN-Chim) using gene-specific primers (Table 1$){ }^{23}$ Similarly, the mCherry gene and click beetle red luciferase gene under the constitutive $P_{\text {IdhL }}$ promoter were successfully transformed into E. coli Nissle 1917 to obtain EcN-Ctrl and EcN-CBRluc, respectively (Table 1). The insertion of the birchgrass pollen chimera along with mCherry, mCherry alone and luciferase gene was confirmed by DNA sequencing. Intracellular production of the chimera from the EcN-Chim was confirmed in immunoblots as a single band corresponding to the calculated mass $(24.43 \mathrm{kD}$ ) of Bet $\mathrm{v} 1$ and two peptides $\mathrm{Phl} \mathrm{p} 1$ and $\mathrm{Phl} \mathrm{p} 5$ detected using monoclonal mouse antibody against Bet $\mathrm{v} 1$ (Supplementary Figure 1a). In contrast, the control strain i.e. E. coli Nissle 1917 expressing only mCherry (EcN-Ctrl) did not show any band with detection antibody against Bet $v 1$ in the immunoblot analysis. Quantification of chimer production by EcN-Chim using ELISA showed that $10^{9}$ CFUs of EcN-Chim produced $1.2 \pm 0.4 \mu \mathrm{g}$ Bet $v 1$ (corresponding to $1.8 \pm 0.6 \mu \mathrm{g}$ of chimer) ${ }^{8}$, whereas EcNCtrl did not produce any chimer. A strong red fluorescence production by EcN-expressing mCherry was detected and quantified by measuring the fluorescence intensity using the SparkControl Magellan plate reader (Supplementary Figure 1b). The bioluminescence produced by the $E$. coli Nissle expressing the

Table 1. Bacterial strains, plasmids, abbriviations for recombinant strains and primers used in this study

\begin{tabular}{|c|c|c|}
\hline a) Strains and plasmids & Description & Reference \\
\hline Lactococcus lactis MG1363 & Plasmid free & 23 \\
\hline Lactobacillus plantarum NCIMB8826 & Originally isolated from human saliva & $\mathrm{NCBI}$ \\
\hline Escherichia coli MC1061 & araD139 $\Delta$ (ara-leu)7696 lacX74 galV galK hsr-hsm rpsL & \multirow{2}{*}{$\begin{array}{l}\text { Invitrogen } \\
46\end{array}$} \\
\hline Escherichia coli Nissle 1917 & Originally isolated from human intestine by Alfred Nissle & \\
\hline E. coli BL21 & Competent $E$. coli for cloning & New England Biolabs \\
\hline pNZ8148 & L. lactis pSH71 replicon, Chloramphenicol resistance; $\mathrm{Cm}^{\mathrm{r}}$ & \multirow{2}{*}{$\begin{array}{l}\text { MoBiTech } \\
8\end{array}$} \\
\hline pHis Parallel 2-Chim & $\begin{array}{l}\text { pHis Parallel } 2 \text { carrying a cDNA of birch-grass pollen chimer consisting Bet } v 1 \\
\text { as scaffold and the immunodominant peptides of Phl p } 1 \text { and Phl p } 5 \text { were } \\
\text { linked to the } N \text { and C-terminus of Bet } v 1 \text { under inducible nisR and nisK } \\
\text { promoter. } \mathrm{Cm}^{r}\end{array}$ & \\
\hline pMEC275 & $\begin{array}{l}\text { pNZ8148 carrying fluorescent mCherry cDNA codon optimized for } L \text {. } \\
\text { plantarum fused to } L \text {. plantarum PldhL (lactate dehydrogenase) constitutive } \\
\text { promoter. } \mathrm{Cm}^{\mathrm{r}}\end{array}$ & $\begin{array}{l}\text { Daniel et al. } \\
\text { in preparation }\end{array}$ \\
\hline pMEC275-Chim & $\begin{array}{l}\text { pNZ8148 carrying codon optimized g-blocks birch-grass pollen chimer } \\
\text { (Phl p 5-Bet v 1-Phl p 1) optimized for E. coli Nissle codon and mCherry as a } \\
\text { reporter gene fused to constitutive PldhL promoter. } \mathrm{Cm}^{\mathrm{r}}\end{array}$ & Present study \\
\hline pMEC256-CBRluc & $\begin{array}{l}\text { pNZ8148 carrying CBRluc CDNA codon optimized for L. plantarum fused to } \\
\text { PldhL promoter. } \mathrm{Cm}^{r}\end{array}$ & \multirow[t]{2}{*}{23} \\
\hline b) Abbreviations for strains & Details & \\
\hline $\mathrm{EcN}$ & Escherichia coli Nissle 1917 original strain - in vitro studies & Present study \\
\hline EcN-Chim & E. coli Nissle expressing birch-grass pollen chimer + mCherry - in vivo studies & Present study \\
\hline EcN-Ctrl & $\begin{array}{l}\text { E. coli Nissle expressing mCherry as a control strain - in vitro and in vivo } \\
\text { studies }\end{array}$ & Present study \\
\hline EcN-CBRluc & E. coli Nissle expressing CBRluc a luciferase gene -in vivo imaging studies & Present study \\
\hline Lp-CBRluc & L. plantarum expressing CBRluc a luciferase gene - in vivo imaging studies & Present study \\
\hline c) Name of primer & Sequence & \\
\hline Phl_p5_Betv1a_Phl_p1_Ec_F (Nco) & ATGATGCCATGGCGTACGCTGCTACTGT & Present study \\
\hline Phl_p5_Betv1a_Phl_p1_Ec_RBS_R (Nco) & CTCATGCCATGGTAATTCCTCCTTTGATTACACCTTCGTGCCTTCCG & Present study \\
\hline Betv1a_Ec_screen_R & ACAGGTTATCACCGTCCAGG & Present study \\
\hline pMEC181_seq_F & ATGACGTGTCTGGGCATATTG & Present study \\
\hline pMEC248_seq_R & TAACAGACAACATCTTCGCTGC & Present study \\
\hline
\end{tabular}

a) All wild type strains and plasmids used in this study. b) abbreviations and details of recombinant strains used in this study. c) Primers used in this study. All primers restriction and modifying enzymes, as well as Q5 DNA polymerase and Taq polymerase were purchased from New England Biolabs (NEB). PCR products were purified using the NucleoSpin Gel and PCR Clean-up Kit (Macherey-Nagel, Germany).

$\mathrm{Cm}^{\mathrm{r}}$ : resistance to chloramphenicol 
luciferase gene (EcN-CBRluc) was detected and quantified using the multimodal in vivo imaging system (IVIS) Lumina XR (Caliper, PerkinElmer) as described previously ${ }^{23}$ (Supplementary Figure 1C).

Intranasal route of application is superior to oral route in preventing allergic poly-sensitization and airway inflammation Allergic airway inflammation. Intranasal pretreatment with EcNChim prior to sensitization with recombinant proteins Bet $v 1, \mathrm{Phl}$ p 1 and Phl p 5 (Fig. 2a) significantly reduced Th2-mediated allergic airway responses i.e. IL-5 and IL-13 in bronchoalveolar lavage (BAL) as compared to other treatment groups especially compared to intranasal-EcN-Ctrl group (Fig. 2b). Lung histology showed reduced cell infiltration and less mucus production in lung in the group treated intranasally with EcN-Chim as compared to any other treated group (Fig. 2c). This reduction was also reflected in the number of eosinophils in BAL (Fig. 2d, e).

Allergen-specific antibody levels in serum and BAL. Intranasal pretreatment of mice with recombinant EcN-Chim led to a significant reduction of allergen-specific IgE antibody levels against Bet $\mathrm{v} 1, \mathrm{Phl} \mathrm{p} \mathrm{1}$, and $\mathrm{Phl} \mathrm{p} 5$ in serum (Fig. 3a). Intranasal pretreatment with EcN-Ctrl led to markedly reduced IgE antibodies against Bet $\mathrm{v} 1$ but not against $\mathrm{Phl} p 1$ or $\mathrm{Phl} p 5$ (Fig. 3a). In contrast, oral pretreatment with neither recombinant nor control EcN led to a significant reduction in allergen-specific serum IgE. Both intranasal groups showed an increased serum allergen-specific $\operatorname{lgG} 2 \mathrm{a} / \mathrm{lgE}$ ratio compared to the polysensitized controls $(P<0.001)$; nevertheless the group treated intranasally with EcN-Chim showed significantly higher allergen-specific $\operatorname{lgG} 2 \mathrm{a} / \mathrm{lgE}$ ratio compared to the group treated intranasally with EcN-Ctrl $(P<0.01)$ (Table 2a). However, serum allergen-specific IgG1 levels did not differ between any of the treated and polysensitized/allergic controls (data not shown). In BAL, allergenspecific IgA antibodies were significantly increased only in mice pretreated intranasally either with EcN-Chim or EcN-Ctrl compared to sensitized controls (Fig. 3b). Oral pretreatment of mice with either recombinant EcN-Chim or EcN-Ctrl showed no change in $\lg \mathrm{A}$ as compared to sensitized control (Fig. 3b).

Intranasal pretreatment with EcN-Chim leads to reduced levels of allergen-specific Th2 cytokine

Allergen-specific Th2 cytokines IL-5 in lung or spleen cell cultures and IL-13 in lung cell cultures were significantly reduced in both intranasally pretreated groups compared to the sensitized controls and compared to orally pretreated groups (Tables 2b, c). Comparing the groups treated intranasally either with EcN-Chim or EcN-ctrl revealed that the suppressive effects on IL-5 and IL-13 production were markedly stronger in the EcN-Chim group. Additionally, increased IL-10 production was observed only in spleen cells stimulated with grass pollen but not with birch pollen in this group (Table 2c). IFN- $\gamma$ levels in spleen cell cultures restimulated with birch pollen extract were significantly reduced in mice treated either orally or intranasally with EcN-Chim (Table 2c).

Intranasal pretreatment with EcN-Chim leads to increased Foxp3, TGF $\beta$, and IL-10 mRNA expression in bronchial lymph nodes (BLNs)

Mice treated intranasally with EcN-Chim showed significantly increased Foxp3, TGF $\beta$ and IL-10 mRNA expression in BLNs as compared to sensitized control and orally treated mice (Fig. $4 a-c$ ). Intranasal treatment with EcN-Chim showed markedly higher levels of Foxp3 and TGF $\beta$ than treatment with EcN-Ctrl (Fig. 4a, b).

E. coli Nissle 1917 induces IL-6 and IL-10 in MLE-12 and MODE-K epithelial cells in vitro

Using confocal microscopy we observed that E. coli Nissle 1917 expressing mCherry was detected at the surface of lung epithelial cells (MLE-12) (Fig. 5a) while in case of the intestinal epithelial cell line (MODE-K), E. coli Nissle was detected both at the surface as well as inside the cell membrane (Fig. 5b).

In vitro stimulation of these cells with the $E$. coli Nissle led to the production of high levels of IL-10 and IL- 6 as compared to unstimulated epithelial cells (Fig. $5 c$, d). There was no statisticaldifference in the production of IFN- $\gamma$, TSLP, and IL-33 between stimulated and unstimulated MLE- 12 and MODE-K cells (Fig. 5c, d).

Intranasally applied E. coli Nissle 1917 transiently migrates through airways and intestine

In order to better understand the migratory behavior of the $\mathrm{EcN}$ in mice after oral and intranasal administration, we studied the spatial and temporal transit of EcN-CBRluc (Bioluminescent EcN) in mice $10 \mathrm{~min}$ and $2 \mathrm{~h}$ after a single oral or intranasal dose. We have used the Lp-CBRluc, a bioluminescent $L$. plantarum as a control because its behavior has been previously studied extensively in the intestine using in vivo imaging. ${ }^{23}$ The bioluminescence signal produced by the EcN-CBRluc and Lp-CBRluc was measured in vivo directly on anesthetized mice and ex vivo, after sacrifice, in the nose, lungs, and gastrointestinal tract of mice using IVIS (Fig. $6 a-c)$. Ten minutes after the oral application of the EcN-CBRluc and Lp-CBRluc strains, bacteria were detected in the small intestine and after $2 \mathrm{~h}$ in cecum (Fig. 6b). After intranasal application, both strains were detected in the nose and lungs during the first $10 \mathrm{~min}$, and after $2 \mathrm{~h}$, strains were also detected in the gut (Fig. 6c).

Mucosal application of E. coli Nissle 1917 leads to short-term persistence compared to the control strain L. plantarum In order to test if the EcN can colonize mucosal surfaces, we treated adult mice either orally or intranasally with EcN-CBRluc and $\mathrm{Lp}$-CBRluc for 4 consecutive days and analyzed the bacterial persistence in vivo (Fig. $7 a-c)$. In the first 4 days of oral administration, a strong bioluminescent signal of both strains was detected only in the gut (Fig. 7b, Supplementary Figure 2a). After intranasal administration, the signal for both bacteria was distributed in the nose, lungs, and gut (Fig. 7c, Supplementary Figure $2 b-d)$. However, the bioluminescent signal in the lungs was lower in intranasally EcN-CBRluc-treated mice as compared to Lp-CBRluc (Supplementary Figure 2c). After day 4, the bioluminescent signal reduced gradually to the background level particularly in the group treated with EcN-CBRluc irrespective of the route of administration and no signal was detected in the nose, lungs or gut after day 6 (Fig. 7b, c; Supplementary Figure 2a-d). On the contrary, the bioluminescent signal in the mice treated orally with $L$. plantarum could still be detected even on day 6 in the gut (Fig. 7b; Supplementary Figure 2a).

To confirm the data on persistence obtained in vivo from the IVIS, we monitored the number of both bacterial strains present in mouse feces and homogenized lung samples collected during the experiment (Fig. 7d, e). Even though the bioluminescent signal for EcN-CBRluc in mice was not detectable after day 6 (Fig. 7b), E. coli Nissle was still present in the feces until day 8 although in very low numbers $\left(10^{1}-10^{2}\right.$ CFU/100 mg of feces). At day 9 , EcN-CBRluc was not detectable anymore in both the orally as well as nasally treated mice (Fig. 7d, e). On the other hand, Lp-CBRluc persisted until day 11 $\left(10^{4}-10^{5} \mathrm{CFU} / 100 \mathrm{mg}\right.$ of feces) in orally treated mice and $\left(10^{3}-10^{4} \mathrm{CFU} / 100 \mathrm{mg}\right.$ of feces) in intranasally treated mice (Fig. $7 d$, e). Although mice received similar bacterial doses $\left(5 \times 10^{8} \mathrm{CFU} / 30 \mu \mathrm{l}\right.$ dose/mouse/day) of bioluminescent $\mathrm{E}$. coli Nissle and $L$. plantarum, the lung samples from days 1 to 4 of mice treated intranasally with ECN-CBRluc showed lower CFU counts $\left(10^{6} \mathrm{CFU} / 100 \mathrm{mg}\right.$ of lung) than in the group treated with Lp-CBRluc $\left(10^{8} \mathrm{CFU} / 100 \mathrm{mg}\right.$ of lung) (Fig. 7e). From day 5, colony counts of both strains declined gradually in the lung. 
PJ Sarate et al.

a

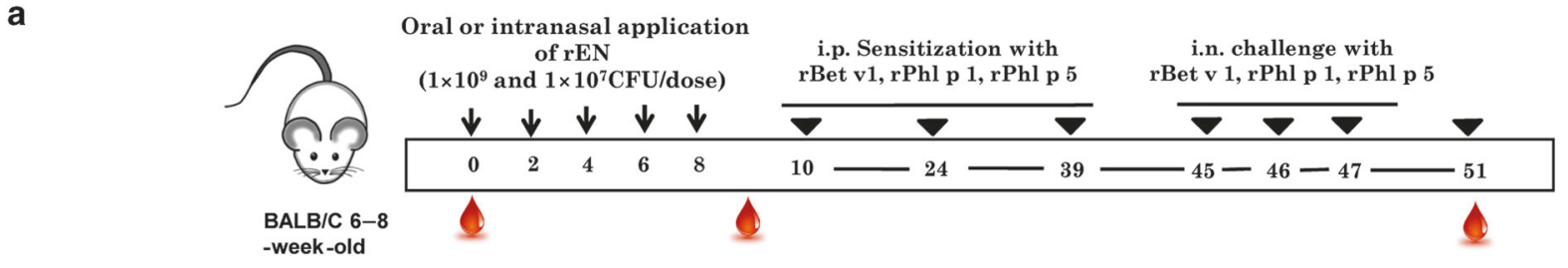

b

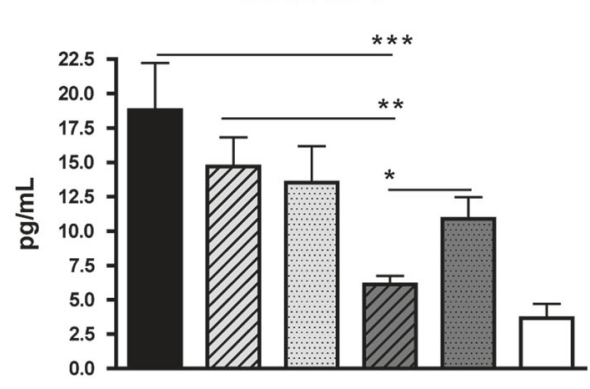

BAL IL-13

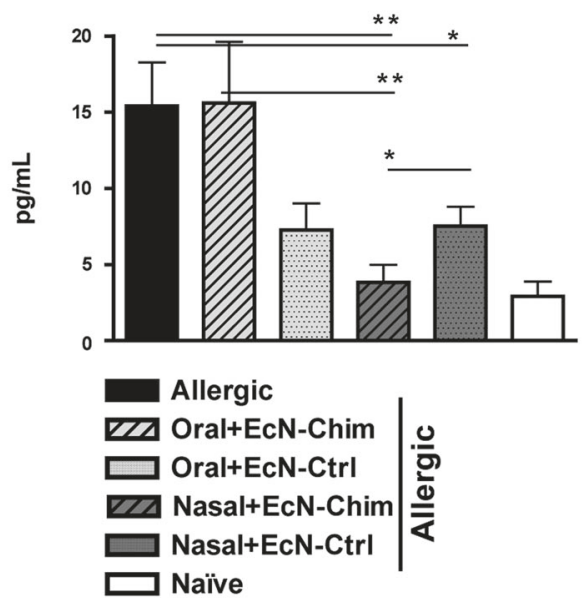

d

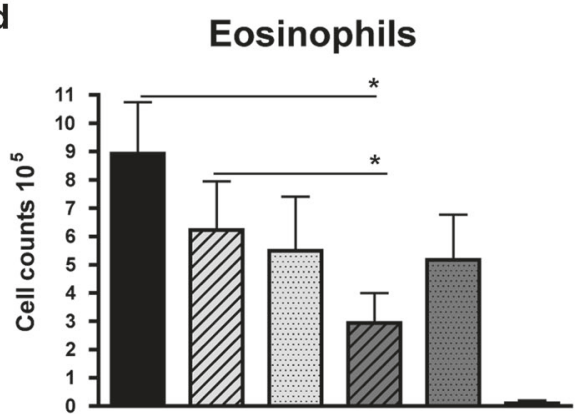

C

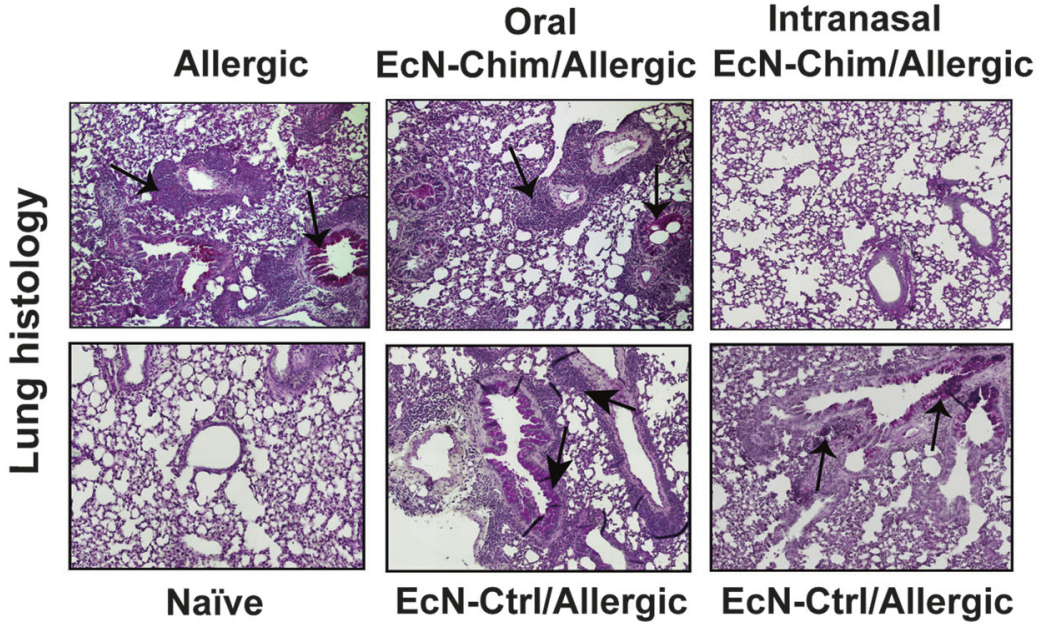

e

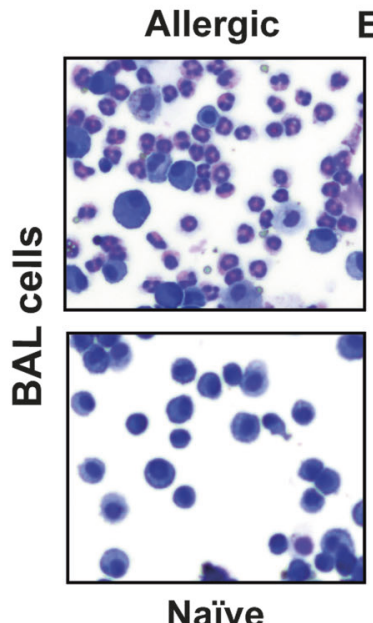

Oral EcN-Chim/Allergic EcN-Chim/Allergic
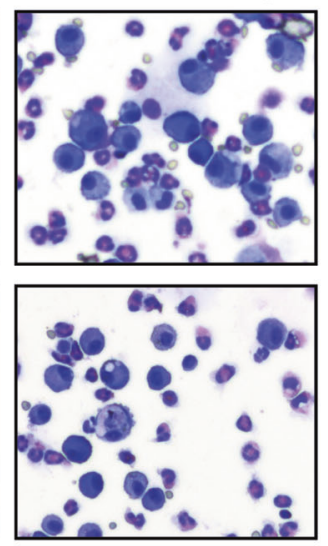

EcN-Ctrl/Allergic
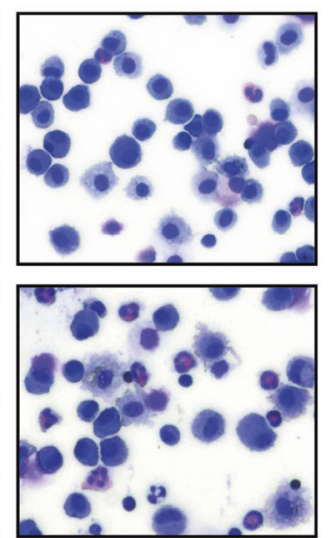

EcN-Ctrl/Allergic

Fig. 2 Intranasal pretreatment with EcN-Chim reduces airway inflammation. a Schematic representation of the pretreatment of recombinant E. coli Nissle expressing birch-grass pollen chimera (EcN-Chim) in a mouse model of poly-sensitization: Female BALB/c mice $(n=5)$ were pretreated with (ECN-Chim) and control E. coli Nissle (ECN-Ctrl) either orally (each $10^{9} \mathrm{CFU}$ in $300 \mu \mathrm{l}$ gavage buffer) or intranasally (each $10^{7} \mathrm{CFU}$ in $30 \mu \mathrm{l}$ in PBS) every second day (days $0,2,4,6,8$ ) followed by intraperitoneal sensitization (days 10, 24, and 39) of a mixture of $5 \mu \mathrm{g} r B e t \mathrm{v} 1$, $5 \mu \mathrm{gPhl} \mathrm{p} \mathrm{1,} \mathrm{and} 5 \mu \mathrm{g} \mathrm{rPhl} \mathrm{p} 5$ adsorbed to aluminium hydroxide and after 1 week an intranasal challenge with $30 \mu \mathrm{l}$ of mixture of $5 \mu \mathrm{g}$ rBet $\mathrm{v}$ $1,5 \mu \mathrm{g} \mathrm{rPhl} \mathrm{p} \mathrm{1,} \mathrm{and} 5 \mu \mathrm{g} \mathrm{rPhl} \mathrm{p} 5$ for 3 consecutive days. Mice were sacrificed $72 \mathrm{~h}$ after the last airway challenge (day 51 ) and blood, bronchoalveolar lavage (BAL), spleens and lungs were collected for further analysis. b IL-5 and IL-13 cytokines in BAL. c Representative lung tissue sections of one mouse per group stained with Periodic Acid Schiff (PAS) (Red; $\times 10$ magnification; scale bars $100 \mu$ m); arrows indicate cell infiltration. d Absolute numbers of eosinophils in BAL. e Representative cytospins of BAL of one mouse per group stained with haematoxylin and eosin (H\&E; $\times 100$ magnification). Allergic: Poly-sensitized control. Oral + EcN-Chim: Oral pretreatment with EcN-Chim prior to polysensitization. Oral + EcN-Ctrl: Oral pretreatment with EcN-Ctrl prior to poly-sensitization. Nasal + EcN-Chim: Intranasal pretreatment with EcNChim prior to poly-sensitizazion. Nasal + EcN-Ctrl: Intranasal pretreatment with EcN-Ctrl prior to poly-sensitization. Naïve: No treatment or poly-sensitization. b, d represent mean \pm SEM from pooled data of two independent experiments (total $n=10$ mice per group). Error bars show mean \pm SEM. ${ }^{*} P<0.05,{ }^{* *} P<0.01,{ }^{* * *} P<0.001$ by Mann-Whitney $U$ - test 
a

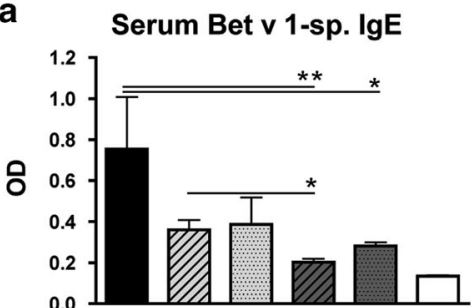

b

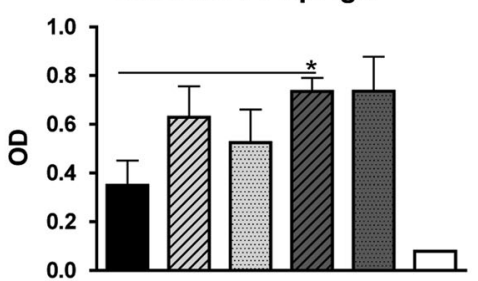

Serum Phl p 1-sp. IgE

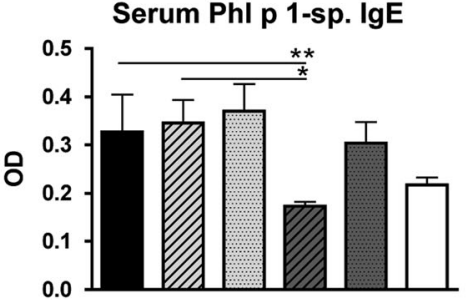

BAL PhI p 1-sp. IgA

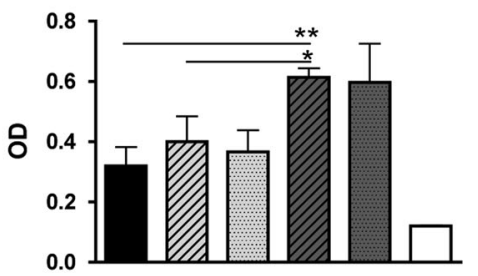

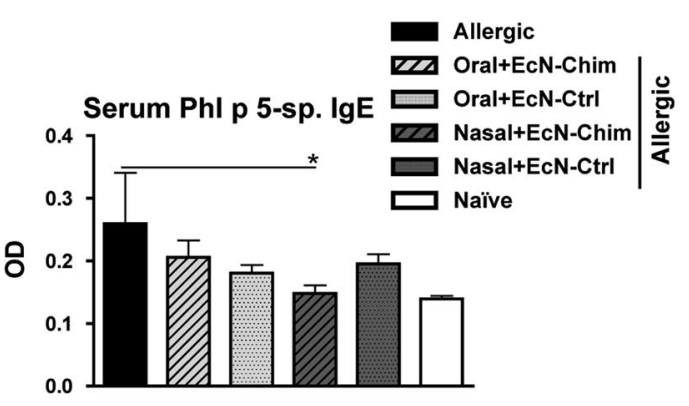

BAL Phl p 5-sp. IgA

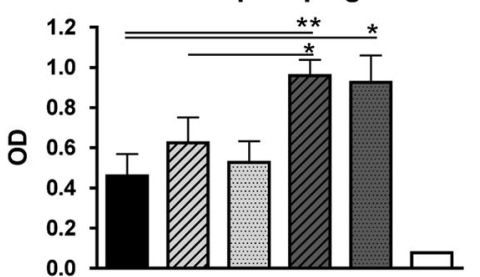

Fig. 3 Intranasal pretreatment with EcN-Chim reduces allergen-specific IgE in serum and induces high levels of IgA in BAL. Mice were treated as indicated in Fig. 2a. Serum samples were obtained from mice blood collected on day 51. Allergen-specific antibody levels in mouse sera or in bronchial lavages (BAL) were determined by ELISA. a Levels of Bet v 1, Phl p 1, Phl p 5-specific serum lgE and $\mathbf{b}$ allergen-specific lgA levels in BAL. $\mathbf{a}, \mathbf{b}$ represents mean \pm SEM from pooled data of two independent experiments (total $n=10$ mice per group). Error bars show mean \pm SEM. ${ }^{*} P<0.05,{ }^{* *} P<0.01$ by Mann-Whitney $U$ - test

Table 2. Antibody response in serum and cytokine response in lung and spleen cells after pretreatment of EcN-Chim or EcN-Ctrl prior to polysensitization and challenge

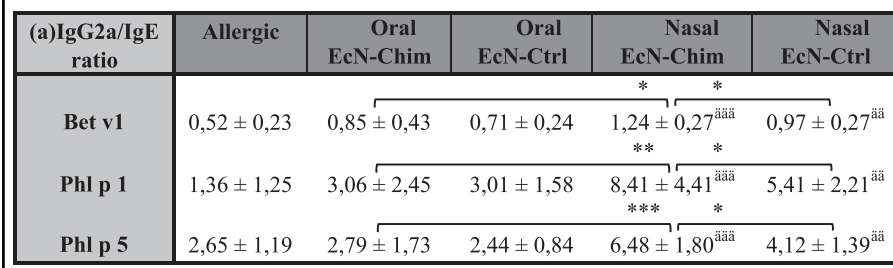

\begin{tabular}{|c|c|c|c|c|c|c|c|c|c|c|}
\hline \multirow{2}{*}{$\begin{array}{c}\text { Cytokines } \\
\text { pg/mL }\end{array}$} & \multicolumn{5}{|c|}{ Birch pollen } & \multicolumn{5}{|c|}{ Grass pollen } \\
\hline & Allergic & $\begin{array}{c}\text { Oral } \\
\text { EcN-Chim } \\
\end{array}$ & $\begin{array}{c}\text { Oral } \\
\text { EcN-Ctrl } \\
\end{array}$ & $\begin{array}{c}\text { Nasal } \\
\text { EcN-Chim } \\
\end{array}$ & $\begin{array}{c}\text { Nasal } \\
\text { EcN-Ctrl } \\
\end{array}$ & Allergic & $\begin{array}{c}\text { Oral } \\
\text { EcN-Chim } \\
\end{array}$ & $\begin{array}{c}\text { Oral } \\
\text { EcN-Ctrl } \\
\end{array}$ & $\begin{array}{c}\text { Nasal } \\
\text { EcN-Chim } \\
\end{array}$ & $\begin{array}{c}\text { Nasal } \\
\text { EcN-Ctrl } \\
\end{array}$ \\
\hline \multicolumn{11}{|l|}{ (b) Lung } \\
\hline \multirow{3}{*}{ IL-5 } & & & & $* * \quad * * *$ & & & & & $* *$ & \\
\hline & $428 \pm 175$ & $312 \pm 160$ & $274 \pm 200$ & $105 \pm 47^{\text {äӓӓ }}$ & $246 \pm 99^{\ddot{a}}$ & $722 \pm 231$ & $437 \pm 194$ & $374 \pm 198^{\mathrm{a}}$ & $255 \pm 84^{\text {äää }}$ & $387 \pm 111^{\text {aีä }}$ \\
\hline & & & & $* * \quad * *$ & & & & & $7^{*}$ & \\
\hline IL-13 & $198 \pm 104$ & $297 \pm 274$ & $225 \pm 220$ & $17 \pm 12^{\text {ääă }}$ & $98 \pm 84^{\mathrm{a}}$ & $838 \pm 324$ & $587 \pm 522$ & $222 \pm 169$ & $167 \pm 103^{\text {aаä̆ }}$ & $225 \pm 116^{\text {äää }}$ \\
\hline \multicolumn{11}{|l|}{ (c) Spleen } \\
\hline \multirow[b]{2}{*}{ IL-5 } & & & & $* *$ & & & & & ** & \\
\hline & $153 \pm 70$ & $153 \pm 87$ & $113 \pm 98$ & $52 \pm 33^{\text {ää }}$ & $117 \pm 92$ & $755 \pm 154$ & $718 \pm 258$ & $587 \pm 246$ & $349 \pm 150^{\text {аäӓ }}$ & $438 \pm 205^{\text {ää }}$ \\
\hline IL-10 & $332 \pm 235$ & $247 \pm 240$ & $177 \pm 160$ & $215 \pm 165$ & $433 \pm 373$ & $2230 \pm 790$ & $2314 \pm 968$ & $2388 \pm 1596$ & $3663 \pm 2303^{\mathrm{a}}$ & $2698 \pm 1616$ \\
\hline IFN $\gamma$ & $1889 \pm 1058$ & $828 \pm 326^{\mathrm{a}}$ & $1424 \pm 1036$ & $988 \pm 351^{\text {ä }}$ & $1229 \pm 873$ & $1711 \pm 1154$ & $1063 \pm 468$ & $1762 \pm 1403$ & $1733 \pm 653$ & $1497 \pm 1048$ \\
\hline
\end{tabular}

Female BALB/c mice were pretreated either orally or intranasally with EcN-Chim and EcN-Ctrl on every second day followed poly-sensitization and challenge with recombinant allergens. Mice were sacrificed $72 \mathrm{~h}$ after the last airway challenge. Serum samples were collected for antibody analysis. Spleens and lungs were collected for cytokine analysis from spleen cell culture and lung cell cultures stimulated with birch and grass pollen allergen. (a) lgG2a/lgE ratio represents in OD. (b) Lung (IL-5, IL-13) and (c) spleen (IL-5, IL-10, IFN $\gamma$ cytokine levels are represented as pg/ml

Data are representative of two independent experiments ( $n=5$ mice per group). Significant difference between allergic control group and treated group is indicated by alphabet (ä) and values are reported as mean \pm SEM. ${ }^{a} P<0.05 ;{ }^{a ̈ a ̈} P<0.01$; äää $P<0.001$. The significant difference between the treated group is indicated by star/asterisk $\left.{ }^{*}\right)$ and values are reported as mean \pm SEM. ${ }^{*} P<0.05 ;{ }^{* *} P<0.01$, ${ }^{* * *} P<0.001$ by Mann-Whitney $U$ test in comparison to polysensitized control 

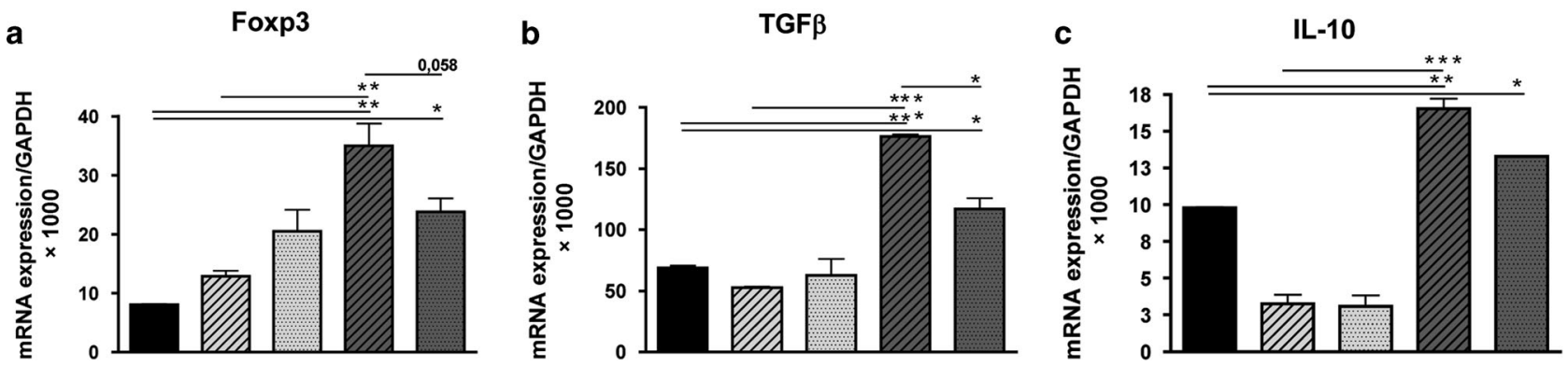

Fig. 4 Intranasal pretreatment with EcN-Chim showed high levels of FOXp3, TGF $\beta$, and IL-10 mRNA in bronchial lymph nodes. Total RNA was extracted from bronchial lymph nodes of mice treated with probiotic bacteria and sensitized/allergic controls at the end of the experiment and reverse-transcribed into CDNA. Expression of FOXp3, TGF $\beta$, and IL-10 mRNA was measured by RT-PCR as described previously. Figure represents the ratio of the target genes to GAPDH, a FOXp3, b TGF $\beta$ and $\mathbf{c}$ IL-10. a-c represents mean \pm SEM from pooled data of two independent experiments (total $n=10$ mice per group). Error bars show mean \pm SEM. ${ }^{*} P<0.05,{ }^{* *} P<0.01,{ }^{* * *} P<0.001$ by one-way ANOVA with Tukey's Multiple Comparison Test
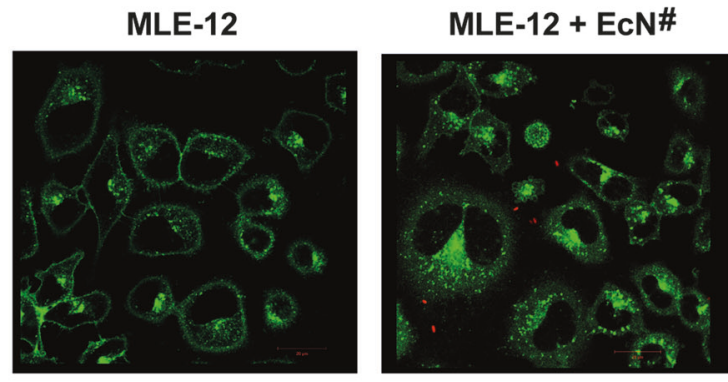

C

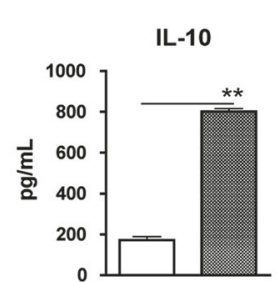

Cytokines in MLE-12

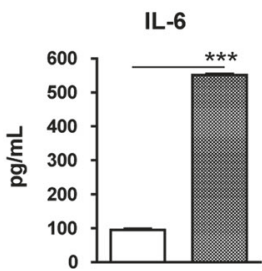

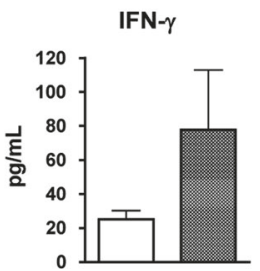

b

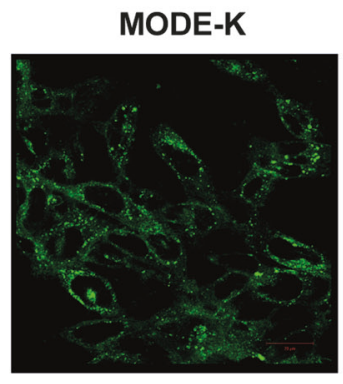

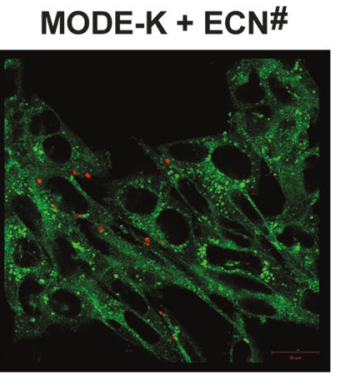

Cytokines in MODE-K

d
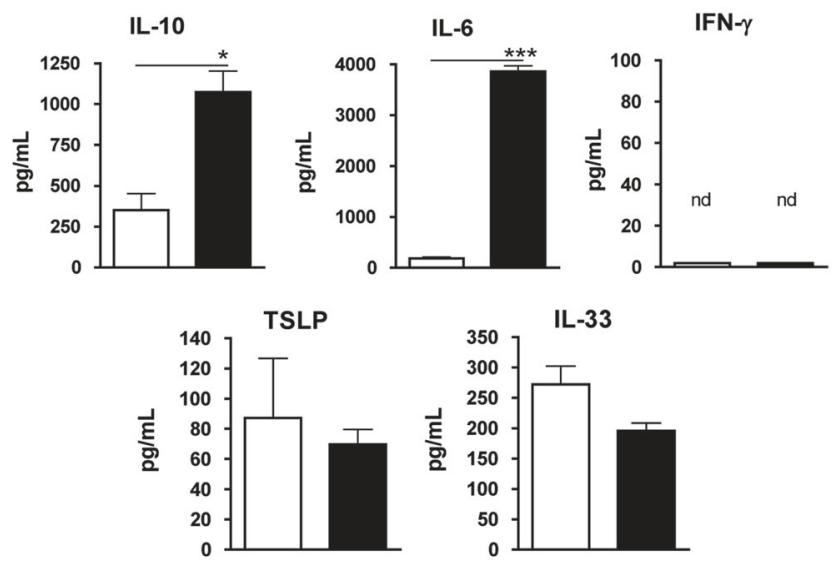

Fig. 5 E. coli Nissle interacts differently with lung and intestinal epithelial cells but induce similar levels of regulatory cytokine IL-10. To understand the interaction of $E$. coli Nissle with epithelial cells, confluent MLE-12 and MODE-K cells were incubated with EcN-expressing mCherry for $2 \mathrm{~h}$ at a bacteria:cell ratio of 10:1. Afterwards, plates were washed with PBS to remove extracellular bacteria and stained with CellMask ${ }^{\mathrm{TM}}$ Plasma Membrane Stain. These cells were then observed under fluorescent microscopy using appropriate filter sets to detect fluorescence of CellMask ${ }^{\mathrm{TM}}$ and $\mathrm{mCherry} .{ }^{49}$ Confocal image showing interaction of fluorescent E. coli Nissle (red) with a MLE-12 cells and $\mathbf{b}$ MODE-K cell (plasma membrane: green). To understand cytokine response upon E. coli Nissle interaction, MLE- 12 and MODE-K cells ( $2.5 \times 10^{4}$ cells/well) were cultured with media only and $10^{7} \mathrm{CFU} / \mathrm{ml}$ of formaline inactivated $\mathrm{EcN}$ followed by incubation at $37^{\circ} \mathrm{C} 5 \% \mathrm{CO}_{2}$ for $48 \mathrm{~h}$. Supernatants were analyzed for IL-6, IL-10, TSLP, IL-33 and IFN- $\gamma$ response in c MLE- 12 cells and d MODE-K cells. Data are representative of two independent experiments (in triplicates). Error bars show mean \pm SEM. ${ }^{*} P<0.05,{ }^{* *} P<0.01,{ }^{* * *} P<0.001$ by Student's $t-$ test. ECN ${ }^{\sharp}$ represents E. coli Nissle expressing mCherry, ND not detected 


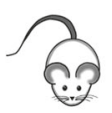

8-week old BALB/c

Oral or Intranasal

pplication of bacteria

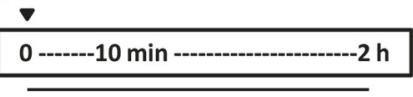

In vivo imaging

b
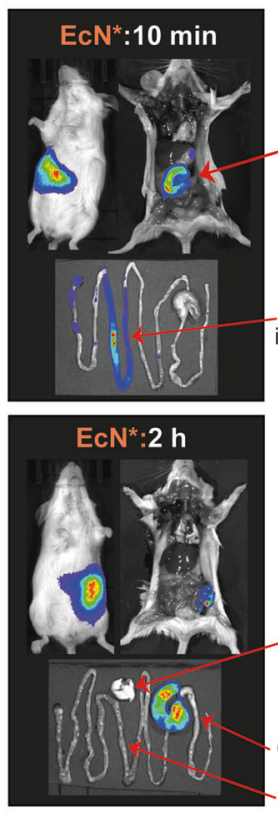

Oral

Gut

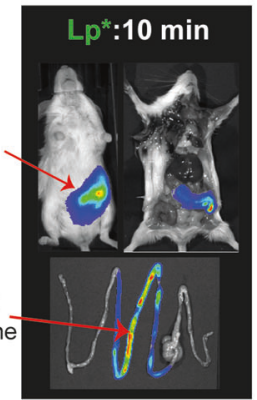

Lung

Cecum

Small

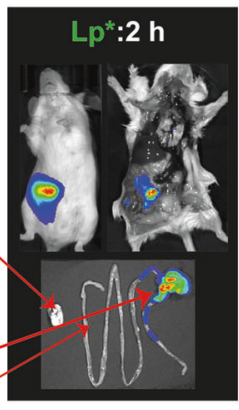

C
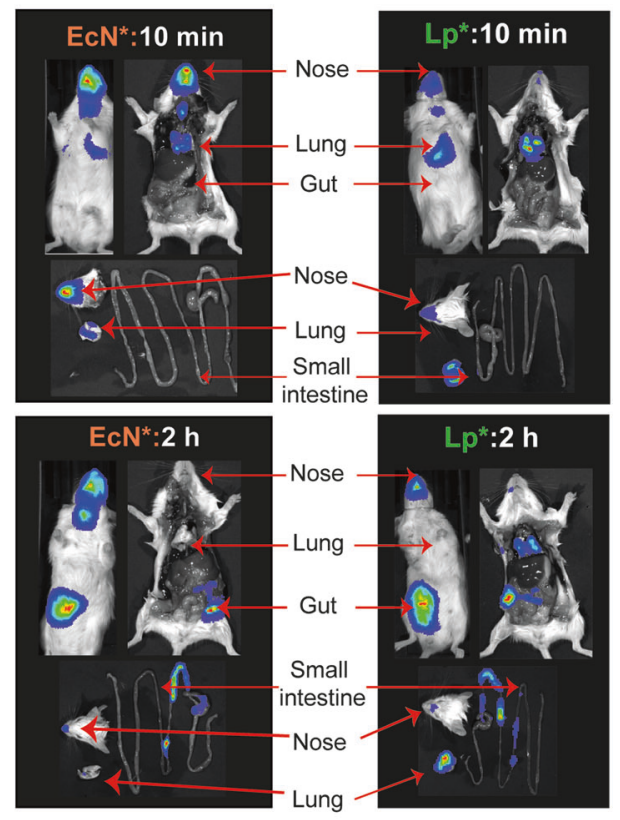

Fig. 6 Intranasally applied $E$. coli Nissle transiently migrates in lungs and gut. Spatial and temporal transit of $E$. coli Nissle was analyzed using the in vivo imaging system (IVIS). L. plantarum was used as control strain. a Schematic representation of the application of EcN-CBRluc and LpCBRluc to study migration of bacteria. Female BALB/C mice were treated either orally $\left(5 \times 10^{10} \mathrm{CFU} / 200 \mu \mathrm{l}\right.$ dose) or intranasally $\left(5 \times 10^{8} \mathrm{CFU} / 30 \mu \mathrm{l}\right.$ dose) with either EcN-CBRluc or Lp-CBRluc. Before in vivo imaging at different time-points, $30 \mathrm{mg} / \mathrm{ml}$ of D-luciferin potassium salt substrate was intragastrically $(200 \mu \mathrm{l} /$ mouse) administered in the groups of mice treated orally and $15 \mu \mathrm{l}$ per nostril in the groups of mice treated intranasally. Mice were anesthetized and immediately bioluminescence imaging of mice was performed using IVIS. b Representative in vivo image of one mouse and its digestive tract at $10 \mathrm{~min}$ and $2 \mathrm{~h}$ after oral application of EcN-CBRluc and Lp-CBRluc. c Representative in vivo image of one mouse and its nose, lungs and gut at $10 \mathrm{~min}$ and $2 \mathrm{~h}$ after intranasal application of EcN-CBRluc and Lp-CBRluc. Organs were collected after sacrifice, and the bioluminescent signal was quantified on intact organs. The intensity of the transcutaneous photon emission is represented as a pseudocolor image. Data are representative of two independent experiments $(n=6$ mice per group). Two mice were sacrificed at each time point. EcN* represents EcN-CBRluc and LP* represents Lp-CBRluc

EcN-CBRluc was not detectable in lungs at day 8 whereas LpCBRluc was still present even at day 10.

No bacteria were detected in the brain of mice treated intranasally with EcN-Chim

To test if bacteria can cross the brain barrier after intranasal treatment with EcN-Chim, homogenized brain samples were analyzed for presence of bacteria using spectrophotometer to measure fluoroscence intensity expressed by $\mathrm{EcN}-\mathrm{Chim}$. No bacteria were detected by spectrophotometer in the brain of mice treated intranasally with EcN-Chim as in brains of untreated controls. These results were confirmed by in vitro colony counts of homogenized brain samples (Supplementary Figure 3)

\section{DISCUSSION}

In this study, we have demonstrated that recombinant $E$. coli Nissle expressing the birch-grass pollen chimera Phl p 5 - Bet v 1$\mathrm{Phl}$ p 1 (EcN-Chim) prevents poly-sensitization in mice. Although the intranasal pretreatment with control strain (EcN-Ctrl) showed certain beneficial effects, the antiallergic effects were more potent in the intranasal group pretreated with recombinant EcN (EcN-
Chim). The intranasal route of application was more effective than the oral route for prevention of systemic sensitization and airway inflammation. Importantly, we have shown that $\mathrm{ECN}$ is a safe probiotic bacterial strain to be used for intranasal antigen delivery as it does not permanently colonize mucosal surfaces.

It has been shown that the intrinsic immunomodulatory properties of probiotic bacteria are strictly strain-specific, where certain strains are potent inducers of Th1 cytokines and others preferably stimulate release of regulatory cytokines such as IL$10 .^{24}$ In allergy treatment, rather than the shift towards Th1 responses, which could lead to autoimmune diseases ${ }^{25}$, suppression of allergen-specific immune responses is a desired goal. IL-10 is considered as an important regulatory cytokine with antiinflammatory properties against allergen-specific Th2 response both in experimental models as well as in humans. ${ }^{26,27}$ Here, we show that EcN stimulates high levels of IL-10, while levels of IL12 p70 are only marginally induced in both spleen cells and BMDCs. The fact that EcN does not stimulate IL-5 in contrast to purified LPS, as previously shown by others ${ }^{28,29}$, further proposes $\mathrm{ECN}$ as a proper strain for mucosal vaccination against allergic poly-sensitization.

In previous studies in mono-sensitized animals we established tolerizing protocols based on allergen-specific or allergen- 
PJ Sarate et al.

a

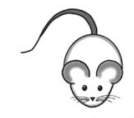

8-week-old

BALB/c

Oral or Intranasal

application of bacteria

$\nabla \nabla \nabla \quad$ Lung and fecal sample collection

$\begin{array}{llllllllll}1 & 2 & 3 & 4 & 5 & 6 & 7 & 8 & \text { - } & \end{array}$

In vivo imaging

b Oral

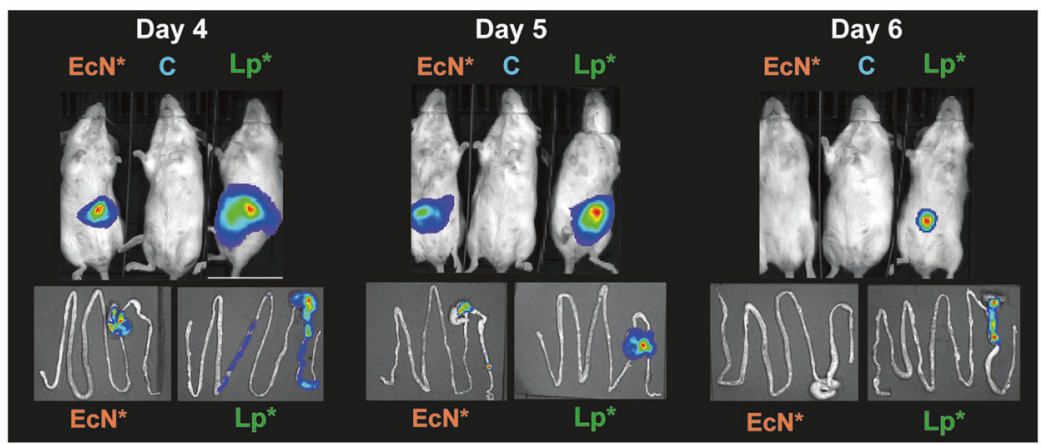

C

\section{Intranasal}

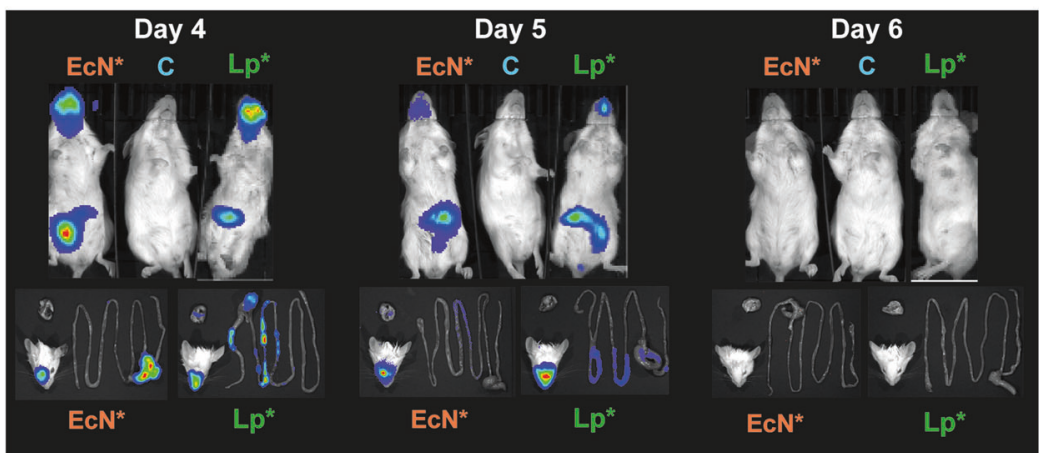

d

Oral: bacterial count in feces

e Intranasal: bacterial count in feces

Intranasal: bacterial count in lung
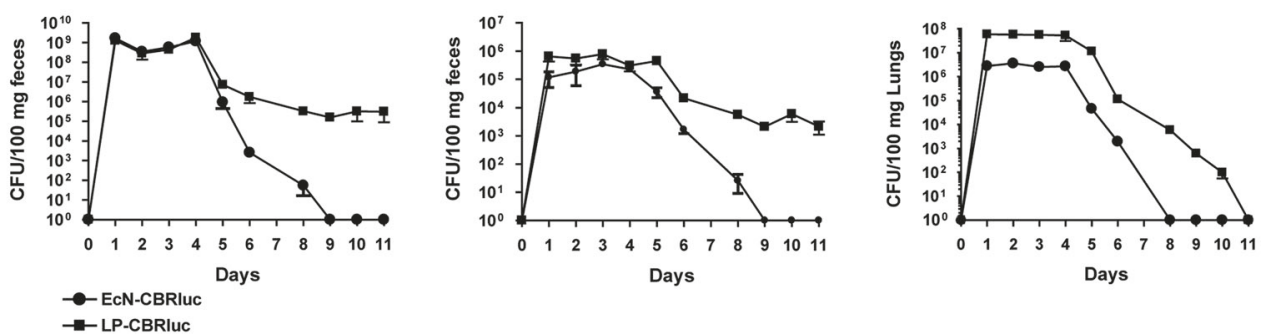

$\rightarrow$-LP-CBRluc

Fig. 7 In vivo and in vitro analysis of persistence of $E$. coli Nissle 1917. Transit of $E$. coli Nissle was studied by analyzing bacterial persistence in specific organs. L. plantarum was used as control strain. a Schematic representation of the experimental protocol. Female BALB/C mice were treated either orally $\left(5 \times 10^{10} \mathrm{CFU} / 200 \mu \mathrm{l}\right.$ dose) or intranasally $\left(5 \times 10^{8} \mathrm{CFU} / 30 \mu \mathrm{l}\right.$ dose) with either EcN-CBRluc or Lp-CBRluc for 4 consecutive days. In vivo bioluminescent signals were analyzed during and after bacterial application until 8 days. Two hours after application and before in vivo imaging $30 \mathrm{mg} / \mathrm{ml}$ of D-luciferin potassium salt substrate was administered intragastrically (200 $\mu \mathrm{l} / \mathrm{mouse})$ in the groups of mice treated orally and $15 \mu \mathrm{l}$ per nostril in group of mice treated intranasally. Mice were anesthetized and immediately bioluminescence imaging of mice was performed using IVIS. Mouse gut (from stomach to rectum), lungs, and head were immediately excised for ex vivo bioluminescence imaging. EcN* represents EcN-CBRluc. Transit of EcN-CBRluc and Lp-CBRluc on days 4, 5 and 6 after b oral application, $\mathbf{c}$ intranasal application. In vivo image contains one representative image of days 4, 5, and 6 containing at least one mouse treated with PBS (middle), EcN-CBRluc (left) and Lp-CBRluc (right). Two mice were sacrificed on each day at $2 \mathrm{~h}$ after bacterial application. Mouse gut, lungs, and head were immediately excised for ex vivo bioluminescence imaging. In the similar parallel experiment the persistence of $E$. coli Nissle and control strain $L$. plantarum in lungs and gut after oral and intranasal application was analyzed by in vitro colony counts in these organs. Mouse feces and lung samples were collected every day (days 1-11). Two mice were sacrificed at each time point to collect lung samples. Black circles represent EcN-CBRluc counts whereas squares represents Lp-CBRluc counts. Averages of the bacterial CFU counts per $100 \mathrm{mg}$ of feces after $\mathbf{d}$ oral application and $\mathbf{e}$ intranasal application of bacteria. e Averages of the bacterial CFU counts per $100 \mathrm{mg}$ of lungs after intranasal application of bacteria. Data are representative of two independent experiments $(n=6$ mice per group). Error bars show mean \pm SEM

nonspecific interventions, such as treatments with recombinant proteins, peptides, probiotics or recombinant probiotics. ${ }^{30}$ It is well recognized that treatment/prevention of poly-sensitization is difficult to achieve. In this respect, we previously tested the use of polypeptides or allergen-chimera constructs as well as probiotic bacteria for prevention of poly-sensitization. ${ }^{7,8,15}$ However both, allergen-specific and nonspecific strategies had certain limitations. Certain wild-type probiotic bacteria acted as transient 
immunomodulators/adjuvants when applied during the sensitization but not all prevented allergy, in particular there was only a mild effect on allergen-specific IgE when applied prior to sensitization. ${ }^{15}$ Using recombinant poly-allergen chimeras for nasal application, allergic poly-sensitization could be reduced but also with limited effects on specific lgE antibodies. ${ }^{8,10}$ With the aim to improve the tolerizing effects of multiallergen constructs, a recombinant EcN expressing the birch-grass pollen chimera was constructed in this study, offering the advantage of protecting the intracellular allergen from enzymatic degradation and guaranteeing sufficient antigen delivery to the respiratory tract as the major treatment target. ${ }^{17}$ To our knowledge this is the first study describing the use of a recombinant probiotic bacteria expressing a poly-allergen construct for prevention of allergy to multiple allergens. Indeed, EcN-Chim showed superiority over the EcN-Ctrl strain as a mucosal treatment tool against poly-sensitization and airway inflammation. Moreover, we show that 100 times less allergen chimer is needed for successful mucosal treatment compared to soluble allergen chimer $^{8}$ supporting the advantage of the use of a bacteria expression/delivery system to minimize the treatment dose. Comparison of the routes of application clearly indicated that the nasal route is more effective than the oral in preventing humoral and cellular immune responses and airway inflammation against birch and grass pollen allergens, thereby supporting the concept of mucosal compartmentalization, ${ }^{31}$ and suggesting that nasal antigen delivery is either less exposed to degradation processes ${ }^{32}$ or allergens are better retained at mucosal surfaces than after oral application as previously stated. ${ }^{33}$ Accordingly we previously showed that lower amounts of antigens are needed for nasal compared to oral treatment/sensitization. ${ }^{34}$

A significant reduction of allergen-specific $\lg \mathrm{E}$ and an increase in allergen-specific $\operatorname{lgG} 2 \mathrm{a} / \mathrm{lgE}$ ratio was preferentially demonstrated after nasal treatment of EcN-Chim compared to polysensitized controls. This seems to be of particular interest as it has been previously shown that IgG2a can inhibit IgE-mediated mast cell degranulation. ${ }^{35}$ Interestingly, despite the increased serum levels of IgG2a, IFN- $\gamma$ levels were not enhanced in spleen cells. A similar finding was described by Adam et al. after intranasal application of EcN in a mouse model of house dust mite allergy. ${ }^{28}$ In our study intranasal tolerance induction with EcN-Chim but less with EcN-Crtl- was further associated with markedly suppressed pulmonary eosinophils and IL-5 and IL-13 levels in BAL and lungs. These results support our previous data showing that recombinant $L$. plantarum expressing Bet $v 1$ allergen but not control $L$. plantarum significantly prevented Bet $v$ 1-specific immune responses. ${ }^{21,22}$

The mechanisms of mucosal tolerance induction have been shown to involve regulatory cells/cytokines as well as $\lg \mathrm{A}$ antibodies. $^{30}$ With respect to tolerance induction with soluble allergen chimeras, we previously showed that Foxp3+ CD4 $+\mathrm{T}$ cells producing TGF- $\beta / \mathrm{IL}-10$ were induced at mucosal sites. ${ }^{8,10}$ Similarly, in the present study, intranasal pretreatment with EcN-Chim and to a lower extend with EcN-Ctrl was associated with increased Foxp3, TGF- $\beta$, and IL-10 mRNA in bronchial lymph nodes. Furthermore, our in vitro studies showed that respiratory and intestinal epithelial cells-as first contact cells at mucosal surfaces ${ }^{36}$ - stimulated with $E$. coli Nissle led to a strong production of IL- 6 and IL-10 cytokines, suggesting that epithelial cells may also contribute to induction of tolerogenic mechanisms by activating dendritic cells which may then migrate to draining lymph nodes and further induce a regulatory/tolerogenic environment. ${ }^{30}$

Furthermore, we detected a marked increase in allergen-specific IgA antibodies in bronchoalveloar lavages. Mucosal IgA production can be induced by T-cell-dependent or T-cell-independent mechanisms of which the latter is primarily stimulated by different cytokines such as IL-6, IL-10, TGF- $\beta$ etc. produced by intestinal epithelial, dendritic, and stromal cells. ${ }^{31}$ That $\lg \mathrm{A}$ antibodies can act as crucial players of protection against allergic airway inflammation was previously shown in a model of ovalbumin allergy using the mucosal adjuvant cholera toxin $B .^{37}$ In a clinical study $\lg A$ has been shown to act as blocking/neutralizing antibodies against IgE-mediated histamine release. ${ }^{38}$ Furthermore, recent clinical studies using the intranasal influenza vaccine (Flumist) showed that intranasal vaccination with live viruses induced $\lg \mathrm{A}$ antibodies with protective properties in both respiratory and gastrointestinal tracts. ${ }^{39}$ Similar pathways might have been induced by the EcN treatment in our in vivo model.

Not only the treatment efficacy but also the behavior of live bacteria at mucosal surfaces might pose an important aspect to consider particularly with regard to safety. We thus studied the migration and persistence of mucosally applied bioluminescent $E$. coli Nissle (EcN-CBRluc) using in vivo imaging. Up to now migration and persistance of these probiotic bacteria have been studied in the gut after oral administration of antibiotic pretreated mice by direct monitoring of bioluminescent bacteria by in vivo imaging ${ }^{40,41}$, while the fate of such bacteria after intranasal administration have not been studied so far. In the present study, we compared the behavior of $E$. coli Nissle after oral and intranasal administration to mice without modifying the normal lung or gut microflora by antibiotic pretreatment. We found that the bioluminescent $E$. coli Nissle strain was exclusively detected in the gut after oral administration as already described. ${ }^{40}$ With regard to intranasal application, we show for the first time the migration of EcNCBRluc in the nose, lung, and gut. One possible explanation for the presence of EcN-CBRluc in the gut after intranasal application could be the reflux reaction of lungs flushing the bacteria back into the nasopharynx as already described. ${ }^{42}$ Furthermore, we studied whether E. coli Nissle showed permanent colonization which could present a risk for sensitization rather than tolerization. We revealed that even after application of high bacterial doses $\left(10^{8} \mathrm{CFU}\right)$ via the nasal route, EcN-CBRluc disappeared within 4 days from lungs as well as from feces of mice after the last application, indicating that $E$. coli Nissle does not permanently colonize the gut nor persists in the lung. Thus, our data suggest that intranasal application results in transient presence of $\mathrm{ECN}$ along the respiratory and intestinal tracts and might activate the immune system at two distinct mucosal compartments leading to effective immunomodulation. With regard to intranasal application of live bacteria in humans, the potential migration of probiotic bacteria into the brain via the olfactory nervous system is often discussed as a safety concern. Here, we show that even after 4 days of consequent application of EcN-Chim (with the same concentration as used for nasal treatment), we did not detect any bacteria in the brain. Our study results are further supported by human studies with live Streptococcus salivarius 24SMB administered by nasal spray for the prevention of acute otitis media in otitis-prone children showing that this application was effective and safe. ${ }^{43}$ Further evidence on biological containment, and tolerability of recombinant probiotic bacteria in humans is currently increasing.

Taken together, our study contributes with novel insights on the use and action of recombinant $E$. coli Nissle expressing a birch-grass pollen chimera to be used as nasal vaccine against allergic polysensitization and airway allergy. Even though we have indications that intranasal application of EcN-Chim can also reduce already established allergic lung inflammation in a therapeutic set-up (data not shown), the effects of this strain were stronger in the prophylactic settings. We further suggest that prevention with such a mucosal tool at an early time point of life would not only counteract the risk of sensitization to many allergens (in patients with positive family history) but also provide bacterial imprinting 
and thereby helping in the establishment of an immunological homeostasis in the postnatal period. ${ }^{44,45}$

\section{MATERIALS AND METHODS}

Wild-type bacteria and growth conditions

Wild-type probiotic bacterial strains Lactobacillus plantarum NCIMB8826, Lactococcus lactis MG1363, Escherichia coli Nissle 1917 (EcN) which is an active component of a microbial preparation (Mutaflor; Ardeypharm GmbH, Herdecke, Germany), E. coli BL-21, and E. coli MC1061 (an expression systems) were used in this study (Table 1). Strains were grown and maintained as previously described. ${ }^{23,46}$

Fixation of bacteria

The fixation of bacteria was performed as described in Supplementary Material online.

Selection of probiotic bacteria according to in vitro stimulatory properties of naïve bone marrow-derived dendritic cells, spleen cells, and human embryonic kidney cells 293 (HEK 293)

BMDCs, spleen cells, and HEK293 cells were isolated and cultured as described previously. ${ }^{15,47}$ All three cell cultures were stimulated with $10^{7} \mathrm{CFU} / \mathrm{ml}$ of L. plantarum, L. lactis and E. coli Nissle 1917 and incubated at $37^{\circ} \mathrm{C}, 5 \% \mathrm{CO}_{2}$ for $48 \mathrm{~h}$. Supernatants were analyzed for cytokine response. See Supplementary Material online for details.

Cloning, transformation, and expression of birch-grass pollen chimera in E. coli Nissle 1917

Three recombinant strains of $E$. coli Nissle expressing (1) birchgrass pollen chimera-mCherry (EcN-Chim), (2) mCherry (EcN-Ctrl) and (3) luciferase (EcN-CBRluc) (Table 1) were constructed. $L$. plantarum with pNZ-CBRluc (Lp-CBRluc) was used as control strain. ${ }^{23}$ Chimera production was analyzed by western blotting and quantified by ELISA using monoclonal mouse anti-Bet $v 1$ IgG antibodies as described previously. ${ }^{8,21}$ Production of mCherry was analyzed by fluorescence intensity under SparkControl Magellan plate reader. Luminescence was measured in bacterial cultures by the in vivo imaging system (IVIS) as described previously. ${ }^{23}$ Strain stability was tested by standard methodology as previously described. ${ }^{23}$ See Supplementary Material online for details.

Antigens and natural extracts

See Supplementary Material online for details.

Animals and ethics statement

In the mouse model of poly-sensitization, female BALB/c mice, 6-8 weeks of age were purchased from Charles River (Sulzfeld, Germany). The animals were maintained under conventional housing conditions and provided with food and water ad libitum. All experiments were approved by the Animal Experimentation Committee of the Medical University of Vienna and by the Federal Ministry of Science and Research (BMWF-66.009/0384-WF/V/3b/2015). For in vivo imaging experiments, 7-week-old female BALB/c mice were purchased from Charles River (St. Germain sur l'Arbresle, France) and were maintained under conventional housing conditions. Animal protocols were approved by the local and national ethics committee and all experiments were done in an accredited establishment (A59107, Institut Pasteur de Lille) according to European guidelines (number 86/609/CEE).

Mouse model of poly-sensitization using recombinant birch and grass pollen allergens

Poly-sensitization was performed as described previously ${ }^{7}$ in mice with recombinant antigens for sensitization as well as challenge
(Fig. 2a). Briefly, sensitization was performed with three intraperitoneal injections (days 10,24, and 39) of a mixture of $5 \mu \mathrm{g}$ rBet $\mathrm{v}$ $1,5 \mu \mathrm{g} \mathrm{rPhl} \mathrm{p} 1$, and $5 \mu \mathrm{g} \mathrm{rPhl} \mathrm{p} 5$ adsorbed to aluminium hydroxide. One week after the last intraperitoneal immunization, mice were anesthetized by $2 \%$ isoflurane in anesthetic induction chamber and challenged intranasally with $30 \mu \mathrm{l}$ of mixture of $5 \mu \mathrm{g}$ rBet $\mathrm{v} 1,5 \mu \mathrm{g} \mathrm{rPhl} \mathrm{p} \mathrm{1,} \mathrm{and} 5 \mu \mathrm{g} \mathrm{rPhl} \mathrm{p} 5$ for 3 consecutive days.

Intranasal and oral application of EcN-Chim/EcN-Ctrl prior to polysensitization

Mice ( $n=5 /$ group) were pretreated on days $0,2,4,6,8$ either orally $\left(1 \times 10^{9} \mathrm{CFU} / 300 \mu \mathrm{l} /\right.$ mice $)$ or intranasally $\left(1 \times 10^{7} \mathrm{CFU} / 30 \mu \mathrm{l} /\right.$ mice) with EcN-Chim or EcN-Ctrl. All mice were then sensitized and challenged according to the poly-sensitization protocol as described above (Fig. 2a). Mice were sacrificed $72 \mathrm{~h}$ after the last challenge and organs were collected for further studies. See Supplementary Material online for details.

Characterization of airway inflammation and allergic polysensitization

BAL samples were collected and analyzed for differential cell counts as previously described. ${ }^{48}$ Excised lungs were fixed with 7.5\% formaldehyde-PBS and paraffin-embedded. Tissue sections were stained with Periodic Acid Schiff (PAS) stain. Allergen-specific antibody levels in mouse sera and BAL and cytokine response in $B A L$, spleens, and lungs were determined by ELISA as previously described. ${ }^{7}$ Expression of FOXp3, TGF $\beta$, and IL-10 mRNA in BLNs were measured by RT-PCR as described previously. ${ }^{15}$ See Supplementary Material online for details.

In vitro interaction studies with $E$. coli Nissle expressing mCherry Confocal imaging. Mouse lung epithelial cells (MLE-12) and mouse intestinal epithelial cells (MODE-K) were cultured at $37^{\circ} \mathrm{C}$ and $5 \% \mathrm{CO}_{2}$ as described in supplementary methods. MLE- 12 cells were kindly provided by Prof. Silvia Knapp (Medical University of Vienna, Austria) and MODE-K cells by Dr. D Kaiserlian (INSERM, Paris, France) respectively. Approximately $1 \times 10^{4}$ cells of MLE-12 and MODE-K cells were grown overnight and then incubated with E. coli Nissle expressing mCherry (EcN-Ctrl) for $2 \mathrm{~h}$ at a bacteria:cell ratio of 10:1. After $2 \mathrm{~h}$ cells were stained and observed under fluorescent microscope (LSM780) as described previously. ${ }^{49}$ See Supplementary Material online for details.

In vitro stimulation of MLE12 and MODE-K cells

MLE-12 and MODE-K cells were harvested and approximately $2.5 \times 10^{4}$ cells were stimulated by $10^{7} \mathrm{CFU} / \mathrm{ml}$ of ECN-expressing mCherry. Supernatants were collected and analyzed for different cytokines.

In vivo bioluminescence imaging of $E$. coli Nissle producing the click beetle luciferase

Mice ( $n=6 /$ group) received either orally $\left(5 \times 10^{10} \mathrm{CFU} / 200 \mu\right.$ l dose per mice) or nasally $\left(5 \times 10^{8} \mathrm{CFU} / 30 \mu \mathrm{l}\right.$ dose per mice) a dose of EcN-CBRluc or Lp-CBRluc and bioluminescence imaging was performed using the multimodal IVIS Lumina XR imaging system (Caliper, PerkinElmer) as described previously. ${ }^{23}$ In experiment 1 , migration of EcN-CBRluc and Lp-CBRluc was studied by in vivo imaging performed $10 \mathrm{~min}$ and $2 \mathrm{~h}$ after bacterial application. In experiment 2 , bacteria were applied for 4 consecutive days and the persistence of the bacteria studied by performing imaging for up to 8 days (Figs. 6, 7 and Supplementary Figure 2). See Supplementary Material online for details.

Evaluation of bacterial persistence in feces and lungs. Fecal and lung samples were collected during and after four consequent application of bacteria until day 11 (Fig. 7) and $100 \mathrm{mg} / \mathrm{ml}$ of fecal and lung samples were mechanically homogenized in PBS. 
Dilutions from respective strains were plated onto either LB agar (E. coli Nissle) or MRS agar (L. plantarum) containing $20 \mu \mathrm{g} / \mathrm{ml}$ chloramphenicol and incubated before enumeration.

In vitro analysis of bacterial presence in brain after intranasal treatment

Presence of $E$. coli Nissle in brain after intranasal application was analyzed in vitro by measuring the fluorescence intensity (for mCherry) and by performing bacterial enumeration in different dilutions of homogenized brains of EcN-Chim and EcN-Ctrl treated mice (Supplementary Figure 3). See Supplementary Material online for details.

\section{Statistical analysis}

Comparison of more than two groups was performed with oneway analysis of variance, followed by Tukey's Multiple Comparison Test unless otherwise specified. Significance between two groups was analyzed using the Mann-Whitney $U$ test (nonparametric) or Student's $t$ - test (parametric) using GraphPad Prism Software, (San Diego, CA). All data are shown as mean \pm SEM. Significant differences were considered at $P<0.05\left({ }^{*}\right), P<0.01\left(^{* *}\right), P<0.001\left(^{* *}\right)$.

\section{ACKNOWLEDGEMENTS}

We gratefully acknowledge funding from the Austrian Science Fund SFB F46 and DK MCCA, OeAD-GmbH grants (FR13/2016) and from the Amadeus partnership Hubert Curein French and Austrian program. We thank the Biolmaging Center of Lille (Frank Lafont) for the use of the IVIS Lumina XR. We gratefully thank Katharina Ambroz, Elke Korb, Karin Baier, Erika Garner-Spitzer, Joshua Tobias, Gwenaëlle Verbrugghe, Jéremy Desramaut, for invaluable technical assistance and helpful discussions.

\section{AUTHOR CONTRIBUTIONS}

U.W. received the grant from FWF, and together with I.S. conceived the study. P.J.S S.H.,C.D., U.W. designed the experiments. P.J.S., C.D., S.P., S.H. performed the experiments. P.J.S., S.H., C.D., U.W. analyzed the data. P.J.S., S.H., C.D., S.P., M.D., C.Z. I.-S., U.W. contributed reagents/materials/analysis tools. P.J.S. and U.W. drafted the manuscript. P.J.S., C.D., I.S., U.W. wrote the manuscript.

\section{ADDITIONAL INFORMATION}

The online version of this article (https://doi.org/10.1038/s41385-018-0084-6) contains supplementary material, which is available to authorized users.

Competing interests: The authors declare no competing interests.

\section{REFERENCES}

1. Yazdanbakhsh, M., Kremsner, P. G. \& van Ree, R. Allergy, parasites, and the hygiene hypothesis. Science 296, 490-494 (2002).

2. Julia, V., Macia, L. \& Dombrowicz, D. The impact of diet on asthma and allergic diseases. Nat. Rev. Immunol. 15, 308-322 (2015).

3. Hua, X., Goedert, J. J., Pu, A., Yu, G. \& Shi, J. Allergy associations with the adult fecal microbiota: analysis of the American Gut Project. EBioMedicine 3, 172-179 (2016)

4. Calderon, M. A., Cox, L., Casale, T. B., Moingeon, P. \& Demoly, P. Multiple-allergen and single-allergen immunotherapy strategies in polysensitized patients: looking at the published evidence. J. Allergy Clin. Immunol. 129, 929-934 (2012).

5. Ciprandi, G. et al. Polysensitization as a challenge for the allergist: the suggestions provided by the Polysensitization Impact on Allergen Immunotherapy studies. Expert Opin. Biol. Ther. 11, 715-722 (2011).

6. Barth, C. et al. Safety aspects of subcutaneous immunotherapy with multiple allergens-a retrospective analysis on polysensitized patients. Eur. Arch. Otorhinolaryngol. 267, 1873-1879 (2010).

7. Hufnagl, K. et al. Intranasal tolerance induction with polypeptides derived from 3 noncross-reactive major aeroallergens prevents allergic polysensitization in mice. J. Allergy Clin. Immunol. 116, 370-376 (2005).

8. Wild, C. et al. A recombinant allergen chimer as novel mucosal vaccine candidate for prevention of multi-sensitivities. Allergy 62, 33-41 (2007).
9. Hufnagl, K. et al. Airway inflammation induced after allergic poly-sensitization can be prevented by mucosal but not by systemic administration of poly-peptides. Clin. Exp. Allergy 38, 1192-1202 (2008).

10. Hoflehner, E. et al. Prevention of birch pollen-related food allergy by mucosal treatment with multi-allergen-chimers in mice. PLoS ONE 7, e39409 (2012).

11. Cano-Garrido, O., Seras-Franzoso, J. \& Garcia-Fruitós, E. Lactic acid bacteria: reviewing the potential of a promising delivery live vector for biomedical purposes. Microb. Cell Fact. 14, 137 (2015).

12. Abrahamsson, T. R. et al. Probiotics in prevention of IgE-associated eczema: a double-blind, randomized, placebo-controlled trial. J. Allergy Clin. Immunol. 119, 1174-1180 (2007).

13. Prescott, S. L. \& Bjorksten, B. Probiotics for the prevention or treatment of allergic diseases. J. Allergy Clin. Immunol. 120, 255-262 (2007)

14. Toh, Z. Q., Anzela, A., Tang, M. L. \& Licciardi, P. V. Probiotic therapy as a novel approach for allergic disease. Front. Pharmacol. 3, 171 (2012).

15. Schabussova, l. et al. Distinctive anti-allergy properties of two probiotic bacterial strains in a mouse model of allergic poly-sensitization. Vaccine 29, 1981-1990 (2011).

16. Repa, A. et al. Mucosal co-application of lactic acid bacteria and allergen induces counter-regulatory immune responses in a murine model of birch pollen allergy. Vaccine 22, 87-95 (2003)

17. Singh, B., Mal, G. \& Marotta, F. Designer probiotics: paving the way to living therapeutics. Trends Biotechnol. 35, 679-682 (2017).

18. Falb, D., Isabella, V. M., Kotula, J. W., Miller, P. F. Bacteria engineered to treat diseases associated with hyperammonemia. US Patent: US9688967B2. Google Patents (2016).

19. Braat, $\mathrm{H}$. et al. A phase I trial with transgenic bacteria expressing interleukin-10 in Crohn's disease. Clin. Gastroenterol. Hepatol. 4, 754-759 (2006).

20. Limaye, S. A. et al. Phase $1 \mathrm{~b}$, multicenter, single blinded, placebo-controlled, sequential dose escalation study to assess the safety and tolerability of topically applied AG013 in subjects with locally advanced head and neck cancer receiving induction chemotherapy. Cancer 119, 4268-4276 (2013).

21. Daniel, $C$. et al. Modulation of allergic immune responses by mucosal application of recombinant lactic acid bacteria producing the major birch pollen allergen Bet v 1. Allergy 61, 812-819 (2006).

22. Schwarzer, M. et al. Neonatal colonization of mice with Lactobacillus plantarum producing the aeroallergen Bet $v 1$ biases towards Th1 and T-regulatory responses upon systemic sensitization. Allergy 66, 368-375 (2011).

23. Daniel, C., Poiret, S., Dennin, V., Boutillier, D. \& Pot, B. Bioluminescence imaging study of spatial and temporal persistence of Lactobacillus plantarum and Lactococcus lactis in living mice. Appl. Environ. Microbiol. 79, 1086-1094 (2013).

24. Licciardi, P. V. \& Tang, M. L. Vaccine adjuvant properties of probiotic bacteria. Discov. Med. 12, 525-533 (2011)

25. Ford, J. G. et al. II-13 and IFN-gamma: interactions in lung inflammation. J. Immunol. 167, 1769-1777 (2001).

26. Akdis, C. A., Blesken, T., Akdis, M., Wüthrich, B. \& Blaser, K. Role of interleukin 10 in specific immunotherapy. J. Clin. Investig. 102, 98-106 (1998).

27. Gad, M. et al. Regulation of the IL-10/IL-12 axis in human dendritic cells with probiotic bacteria. Fems Immunol. Med. Microbiol. 63, 93-107 (2011).

28. Adam, E. et al. Probiotic Escherichia coli Nissle 1917 activates DC and prevents house dust mite allergy through a TLR4-dependent pathway. Eur. J. Immunol. 40, 1995-2005 (2010).

29. Grozdanov, L. et al. A single nucleotide exchange in the wzy gene is responsible for the semirough $\mathrm{O} 6$ lipopolysaccharide phenotype and serum sensitivity of Escherichia coli strain Nissle 1917. J. Bacteriol. 184, 5912-5925 (2002).

30. Smole, U., Schabussova, I., Pickl, W. F. \& Wiedermann, U. Murine models for mucosal tolerance in allergy. Semin. Immunol. 30, 12-27 (2017).

31. Lycke, N. Recent progress in mucosal vaccine development: potential and limitations. Nat. Rev. Immunol. 12, 592 (2012).

32. Kim, S.-H. \& Jang, Y.-S. The development of mucosal vaccines for both mucosal and systemic immune induction and the roles played by adjuvants. Clin. Exp. Vaccin. Res. 6, 15-21 (2017).

33. Rimmer, J., Santos, C., Yli-Panula, E., Noronha, V. \& Viander, M. Clinical and laboratory studies of the fate of intranasal allergen. PLOS ONE 10, e0127477 (2015).

34. Wiedermann, U. et al. Suppression of antigen-specific T- and B-cell responses by intranasal or oral administration of recombinant bet $\mathrm{v} 1$, the major birch pollen allergen, in a murine model of type I allergy. J. Allergy Clin. Immunol. 103, 1202-1210 (1999).

35. Philips, J. R. et al. The effectiveness of different rat IgG subclasses as IgE-blocking antibodies in the rat basophil leukaemia cell model. Immunol. Cell Biol. 77 121-126 (1999).

36. Espinosa, V. \& Rivera, A. First line of defense: innate cell-mediated control of pulmonary aspergillosis. Front. Microbiol. 7, 272 (2016).

37. Smits, H. H. et al. Cholera toxin B suppresses allergic inflammation through induction of secretory lgA. Mucosal Immunol. 2, 331-339 (2009). 
144

38. Platts-Mills, T. A., von Maur, R. K., Ishizaka, K., Norman, P. S. \& Lichtenstein, L. M. $\lg A$ and $\lg G$ anti-ragweed antibodies in nasal secretions. Quantitative measurements of antibodies and correlation with inhibition of histamine release. J. Clin. Invest. 57, 1041-1050 (1976).

39. Carter, N. J. \& Curran, M. P. Live attenuated influenza vaccine (FluMist(R); Fluenz): a review of its use in the prevention of seasonal influenza in children and adults. Drugs 71, 1591-1622 (2011).

40. Loessner, $\mathrm{H}$. et al. Drug-inducible remote control of gene expression by probiotic Escherichia coli Nissle 1917 in intestine, tumor and gall bladder of mice. Microbes Infect. 11, 1097-1105 (2009).

41. Remer, K. A. et al. Split immune response after oral vaccination of mice with recombinant Escherichia coli Nissle 1917 expressing fimbrial adhesin K88. Int. J. Med. Microbiol. 299, 467-478 (2009).

42. Southam, D. S., Dolovich, M., O'Byrne, P. M. \& Inman, M. D. Distribution of intranasal instillations in mice: effects of volume, time, body position, and anesthesia. Am. J. Physiol. Lung Cell. Mol. Physiol. 282, L833-L839 (2002).

43. Marchisio, $P$. et al. Streptococcus salivarius $24 \mathrm{SMB}$ administered by nasal spray for the prevention of acute otitis media in otitis-prone children. Eur. J. Clin. Microbiol. Infect. Dis. 34, 2377-2383 (2015).

44. Ohland, C. L. \& Jobin, C. Microbial activities and intestinal homeostasis: a delicate balance between health and disease. Cell. Mol. Gastroenterol. Hepatol. 1, 28-40 (2015).

45. Shukla, S. D., Budden, K. F., Neal, R. \& Hansbro, P. M. Microbiome effects on immunity, health and disease in the lung. Clin. Trans. Immunol. 6, e133 (2017).

46. Bickert, T. et al. Probiotic Escherichia coli Nissle 1917 suppresses allergen-induced Th2 responses in the airways. Int. Arch. Allergy Immunol. 149, 219-230 (2009).
47. Schabussova, I. et al. Perinatal maternal administration of Lactobacillus paracasei NCC 2461 prevents allergic inflammation in a mouse model of birch pollen allergy. PLOS ONE 7, 6 (2012).

48. Winkler, B. et al. Mucosal tolerance as therapy of type I allergy: intranasal application of recombinant Bet $v 1$, the major birch pollen allergen, leads to the suppression of allergic immune responses and airway inflammation in sensitized mice. Clin. Exp. Allergy 32, 30-36 (2002).

49. Grimm, V., Gleinser, M., Neu, C., Zhurina, D. \& Riedel, C. U. Expression of fluorescent proteins in bifidobacteria for analysis of host-microbe interactions. Appl. Environ. Microbiol. 80, 2842-2850 (2014).

(i) Open Access This article is licensed under a Creative Commons adaptation, distribution and reproduction in any medium or format, as long as you give appropriate credit to the original author(s) and the source, provide a link to the Creative Commons license, and indicate if changes were made. The images or other third party material in this article are included in the article's Creative Commons license, unless indicated otherwise in a credit line to the material. If material is not included in the article's Creative Commons license and your intended use is not permitted by statutory regulation or exceeds the permitted use, you will need to obtain permission directly from the copyright holder. To view a copy of this license, visit http://creativecommons. org/licenses/by/4.0/.

(c) The Author(s) 2018 